\title{
Orbital and spin magnetic moments of transforming one-dimensional iron inside metallic and semiconducting carbon nanotubes
}

\begin{abstract}
Antonio Briones-Leon, Paola Ayala, Xianjie Liu, Kazuhiro Yanagi, Eugen Weschke, Michael
\end{abstract}
Eisterer, Hua Jiang, Hiromichi Kataura, Thomas Pichler and Hidetsugu Shiozawa

\section{Linköping University Post Print}

\section{Tweet}

N.B.: When citing this work, cite the original article.

Original Publication:

Antonio Briones-Leon, Paola Ayala, Xianjie Liu, Kazuhiro Yanagi, Eugen Weschke, Michael Eisterer, Hua Jiang, Hiromichi Kataura, Thomas Pichler and Hidetsugu Shiozawa, Orbital and spin magnetic moments of transforming one-dimensional iron inside metallic and semiconducting carbon nanotubes, 2013, Physical Review B. Condensed Matter and Materials Physics, (87), 19.

http://dx.doi.org/10.1103/PhysRevB.87.195435

Copyright: American Physical Society http://www.aps.org/

Postprint available at: Linköping University Electronic Press http://urn.kb.se/resolve?urn=urn:nbn:se:liu:diva-94606 


\title{
Orbital and spin magnetic moments of transforming one-dimensional iron inside metallic and semiconducting carbon nanotubes
}

\author{
Antonio Briones-Leon,,${ }^{1 *}$ Paola Ayala, ${ }^{1}$ Xianjie Liu, ${ }^{2}$ Kazuhiro Yanagi, ${ }^{3}$ Eugen Weschke, ${ }^{4}$ Michael Eisterer, ${ }^{5}$ Hua Jiang, ${ }^{6}$ \\ Hiromichi Kataura, ${ }^{7}$ Thomas Pichler, ${ }^{1}$ and Hidetsugu Shiozawa ${ }^{1}$ \\ ${ }^{1}$ Faculty of Physics, University of Vienna, Strudlhofgasse 4, 1090 Vienna, Austria \\ ${ }^{2}$ Department of Physics, Chemistry and Biology (IFM), Linköping University, 58333 Linköping, Sweden \\ ${ }^{3}$ Department of Physics, Tokyo Metropolitan University, Hachiouji, Tokyo 192-0397, Japan \\ ${ }^{4}$ Helmholtz-Zentrum Berlin für Materialien und Energie, Wilhelm-Conrad-Röntgen-Campus BESSY II, Albert-Einstein-Str. 15, \\ 12489 Berlin, Germany \\ ${ }^{5}$ Atominstitut, Vienna University of Technology, Stadionallee 2, 1020 Vienna, Austria \\ ${ }^{6}$ NanoMaterials Group, Department of Applied Physics and Center for New Materials, Aalto University, P.O. Box 15100, FI-00076 Aalto, \\ Espoo, Finland \\ ${ }^{7}$ National Institute of Advanced Industrial Science and Technology (AIST), Tsukuba 305-8565, Japan \\ (Received 31 August 2012; revised manuscript received 3 May 2013; published 20 May 2013)
}

\begin{abstract}
The orbital and spin magnetic properties of iron inside metallic and semiconducting carbon nanotubes are studied by means of local x-ray magnetic circular dichroism (XMCD) and bulk superconducting quantum interference device (SQUID). The iron-nanotube hybrids are initially ferrocene filled single-walled carbon nanotubes (SWCNT) of different metallicities. We show that the ferrocene's molecular orbitals interact differently with the SWCNT of different metallicities with no significant XMCD response. At elevated temperatures the ferrocene molecules react with each other to form cementite nanoclusters. The XMCD at various magnetic fields reveal that the orbital and/or spin magnetic moments of the encapsulated iron are altered drastically as the transformation to the 1D clusters takes place. The orbital and spin magnetic moments are both found to be larger in filled semiconducting nanotubes than in the metallic sample. This could mean that the magnetic polarization of the encapsulated material depends on the metallicity of the tubes. From a comparison between the iron $3 \mathrm{~d}$ magnetic moments and the bulk magnetism measured by SQUID, we conclude that the delocalized magnetisms dominate the magnetic properties of these $1 \mathrm{D}$ hybrid nanostructures.
\end{abstract}

DOI: 10.1103/PhysRevB.87.195435

PACS number(s): 75.75.-c, 75.20.-g, 73.22.-f, 78.70.Dm

\section{INTRODUCTION}

The extraordinary electronic and mechanical properties of single-walled carbon nanotubes (SWCNT) make them excellent candidates as building components for micro- and nanodevices. The magnetic properties of SWCNT are highly anisotropic due to their unique 1D nanostructure. The diamagnetic nature of carbon nanotubes (CNTs) was predicted theoretically for semiconducting and metallic nanotubes. ${ }^{1}$ Later, this was observed experimentally. ${ }^{2}$ It was reported that the electronic band structure of SWCNTs is altered at applied magnetic fields parallel or perpendicular to the tube axis due to their anisotropy, leading to novel magnetic, magnetotransport and magnetooptical properties ${ }^{3}$ elemental for device applications such as high density magnetic recording media. Theoretical and experimental studies have shown that mechanical, chemical, and electronic properties can be tuned by different means of functionalization. ${ }^{4}$ In particular, endohedral functionalization or filling of carbon nanostructures with molecules has become a promising means to change or even control the electronic and magnetic properties of these hybrid nanostructures. ${ }^{5}$ Since the first observation of peapods, i.e., SWCNT accommodating buckminster fullerenes, ${ }^{6}$ various molecules and compounds including metallocenes and salts have been encapsulated in the hollow core of CNTs. ${ }^{7-17}$ Encapsulated in CNTs, the filling material is protected against oxidation by the rolled up graphene layer. Suggested applications of such materials are magnetorecording devices ${ }^{18}$ and nanoscale thermometers for biological purposes. ${ }^{19}$
Previous studies on multiwalled carbon nanotubes (MWCNT) encapsulating magnetic nanoparticles (Fe, Ni, or Co) grown by chemical vapor deposition (CVD) have shown the magnetic coercivity in contrast to those without catalytic particles inside. ${ }^{18,20-23}$ A study of magnetic properties of the so-called HiPco nanotubes showed a superparamagnetic behavior, which was attributed to the remaining catalytic particles. ${ }^{24}$ A ferromagnetic behavior was observed in Fe@SWCNT even at room temperature, which was explained as a result of a high degree of Fe filling into the nanotubes and the interaction between the Fe nanowires in the bundles of SWCNT. ${ }^{25}$ A theoretical study showed that the local magnetic moment of Fe nanowires encapsulated in SWCNT depends on the size of the Fe nanoparticles, due to the interaction between the particle and the nanotube. ${ }^{26}$ It was shown experimentally that the encapsulation of Fe in SWCNT strongly alters the spin magnetic moment and the magnitude of magnetic anisotropy energy. $^{27}$

Another attempt to alter the magnetic properties of carbon nanotubes is via the filling of SWCNT with endohedral metallofullerenes. ${ }^{28}$ Significant changes in magnetic moment of SWCNT encapsulating metallofullerenes $\left(\mathrm{Gd} @ \mathrm{C}_{82}\right.$ and Dy@ $\left.\mathrm{C}_{82}\right)$ were found at low temperature $(10 \mathrm{~K})$, attributed to the charge transfer from the metallofullerene to the SWCNT. ${ }^{10,29}$ In addition, SWCNT filled with magnetic salts such as $\mathrm{ErCl}_{3}$ were studied. The magnetization of purified empty SWCNT was measured and found to be much lower than in $\mathrm{ErCl}_{3}$ nanowires grown into the SWCNT where the 
magnetization values are the same as the bulk anhydrous $\mathrm{ErCl}_{3} \cdot{ }^{12,30}$

In recent years, metallocene filled and especially ferrocene filled carbon nanotubes $\left(\mathrm{FeCp}_{2} @ \mathrm{SWCNT}\right)$ have been widely studied both experimentally and theoretically. Their electronic properties can be modified via filling followed by the transformation of the molecules inside into inner tubes and cementite clusters $^{14,31}$ utilizing a nanochemical reaction. By the decomposition of the $\mathrm{FeCp}_{2}$ inside the nanotubes, encapsulated $\mathrm{Fe}$ nanowire can be formed. Such Fe@SWCNT were reported to show a higher magnetization than the FeCp $2 @ \mathrm{SWCNT}$ and the pristine SWCNT, and exhibit ferromagnetism and superparamagnetism at different temperatures. ${ }^{13}$

Superconducting quantum interference device (SQUID) is used to measure the "bulk" magnetic character of these magnetic 1D nanostructures. X-ray magnetic circular dichroism (XMCD) spectroscopy allows us to unravel the magnetic states of specified atomic orbitals in compounds and to identify the spin and orbital magnetic moments. ${ }^{32}$ Hence, XMCD has become a powerful tool for studying the magnetization of a variety of magnetic materials, even in the paramagnetic phase. ${ }^{33}$ In the study of filled SWCNT hybrids it was applied to investigate the local magnetic properties of metallofullerene filled and $\mathrm{ErCl}_{3}$ filled SWCNT. ${ }^{10,12}$ Yet, none of these studies was done as a function of the metallic character of the SWCNT.

In the present work, we study the local magnetic properties of iron hosted in high-purity metallic and semiconducting SWCNT samples. The iron was initially introduced inside SWCNT in the form of ferrocene $\left(\mathrm{FeCp}_{2}\right)$ and transformed to $1 \mathrm{D}$ cementite clusters in DWCNT via a nanochemical pyrolysis. We show from x-ray absorption spectroscopy (XAS) that in the filled starting material the molecular orbital states of ferrocene interact differently with the states of the SWCNT with different metallicities while we observe no significant $\mathrm{XMCD}$ response. In contrast, the nanotubes filled with cementite clusters exhibit enhanced XMCD signals. The orbital $\left(m_{L}\right)$ and spin $\left(m_{S}\right)$ magnetic moments, evaluated by using the sum rules, ${ }^{34,35}$ are well fitted by the modified Langevin function. Both, the orbital and spin magnetic moments are found to be larger in filled semiconducting nanotubes than in the metallic sample. This could mean that the magnetic polarizations of the encapsulated material are dependent on the metallicity of the SWCNT.

Semiconducting and metallic pristine SWCNT show a diamagnetic response in SQUID measurements. Ferromagnetism is observed for the iron carbide filled metallic SWCNT and paramagnetism for the filled semiconducting tubes. The coercivity depends on the degree of filling. Much larger positive magnetic moments per iron molecular unit observed by SQUID mean that the delocalized and/or possibly non-iron magnetic polarizations dominate the magnetism of the encapsulated materials, in association with the local moment of the $\mathrm{Fe} 3 \mathrm{~d}$ state and the diamagnetism of the SWCNT.

\section{EXPERIMENTAL METHODS}

\section{A. Samples preparation}

The metallicity-sorted SWCNT samples used in our experiments were synthesized by the arc-discharge process followed by purification, sorting, and film preparation as reported elsewhere. ${ }^{36}$ The high purity of the SWCNT was confirmed by the X-ray photoemission and C 1s x-ray absorption (XAS) observations, as well as the 1D characters at the valence-band region, i.e., van Hove singularities (vHS) and TomonagaLuttinger-liquid behavior, which appear only in SWCNT with extremely high purity. ${ }^{37-39}$ Metallic, semiconducting, and mixed nanotube samples were filled with ferrocene as described elsewhere. ${ }^{40,41}$ The effective filling was estimated by converting them into double-walled (DW) CNTs by annealing in vacuum at $500{ }^{\circ} \mathrm{C}$ for several hours. Using Raman spectroscopy with a $599 \mathrm{~nm}$ excitation wavelength, the radial breathing mode (RBM) of the original filled SWCNT was observed at a wave number of $159 \mathrm{~cm}^{-1}$ corresponding to an average diameter of $\sim 1.5 \mathrm{~nm}$. After the annealing treatment, an RBM was observed at around $333 \mathrm{~cm}^{-1}$, which corresponds to an inner tube with an average diameter $\sim 0.75 \mathrm{~nm}$ of the DW structure.

Transmission electron microscopy (TEM) was performed using an aberration-corrected JEOL-2200FS FEG TEM operated at $80 \mathrm{kV}$. The samples were sonicated in acetone and the suspensions were cast onto a holey carbon grid. Figure 1 reveal the metal nanoclusters created after the annealing in both the semiconducting [Figs. 1(a)-1(c)] and metallic nanotubes [Figs. 1(d)-1(f)]. The high-resolution micrographs in panels (c), (e), and (f) exhibit inner tube structures being formed in the original tubes, as indicated by the arrows in panel (c).

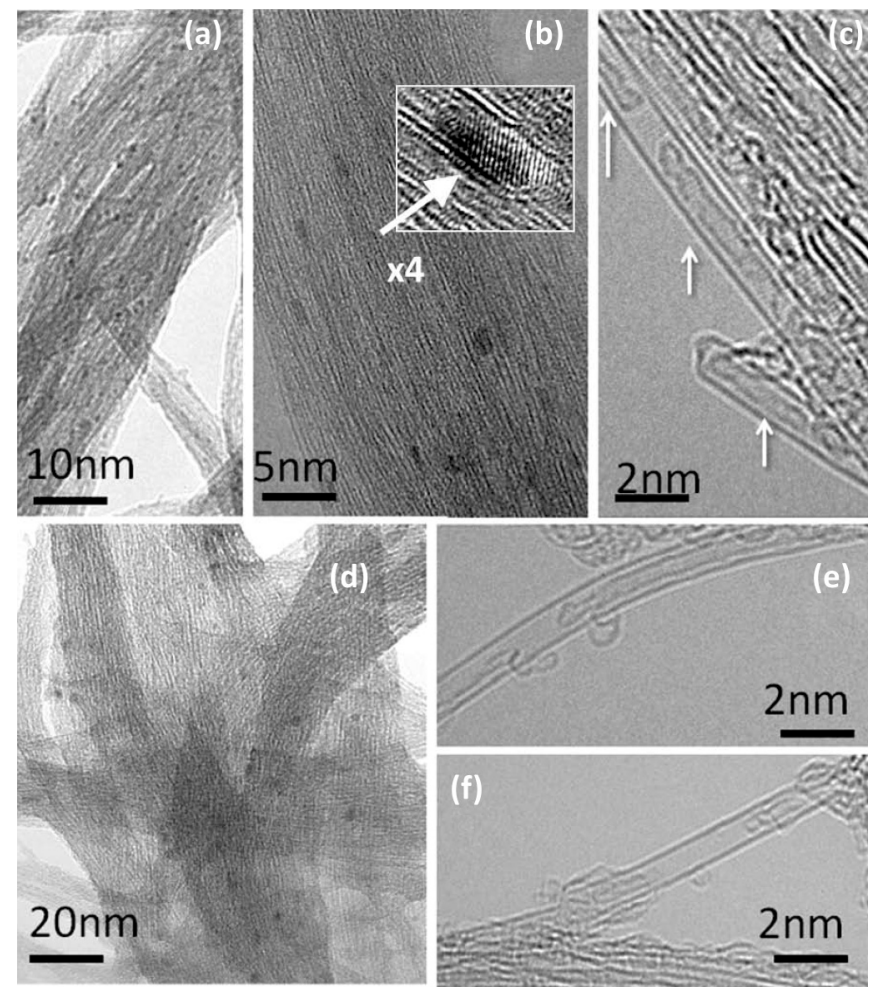

FIG. 1. TEM characterization of the samples. Overall low magnification (a), (b) and intermediate magnification (c) images of the semiconducting-tubes sample. The inset in (b) is a high magnification micrograph of one of the Fe clusters in the main panel. Corresponding overall low magnification (d) and intermediate magnification (e), (f) images of the metallic-tubes sample after annealing. 
The lower magnification micrographs in panels (a), (b), and (d) show the dimension of the encapsulated metal clusters. The inset in panel (b) clearly shows that these clusters have a diameter comparable to the diameter corresponding to the encapsulating tubes, and they are elongated to around double their width. As shown in the previous TEM observations on similar samples, ${ }^{14}$ the cementite nature of the clusters has already been confirmed, which catalyses the inner tube formation. This is consistent with the chemical characterization via XAS in the present study. These results prove that the ferrocene is encapsulated in the hollow space of the nanotubes and successively transformed into cementite clusters by annealing.

\section{B. Measurement of magnetic properties}

Fe 2p XAS and XMCD measurements were carried out at the variable polarization undulator beamline UE46-PGM-1 at the BESSY II (Helmholtz-Zentrum Berlin) synchrotron facility. Circular polarization dependent XAS were obtained by measuring the sample drain current with the photon helicity parallel $\left(\mu^{+}\right)$or antiparallel $\left(\mu^{-}\right)$to the sample magnetization. The experimental end-station of this beamline allows cooling the sample down to $5 \mathrm{~K}$ at a magnetic field up to $6 \mathrm{~T}$. XAS spectra were taken at the $\mathrm{L}_{2,3}$ edge of $\mathrm{Fe}$, with photon energies ranging from 680 to $750 \mathrm{eV}$. The base pressure in the measurement chamber was kept below $5 \times 10^{-10}$ mbar. A heating station in the preparation chamber was used to in-situ anneal the FeCp $\mathrm{p}_{2} @ \mathrm{SWCNT}$ bucky papers at $500{ }^{\circ} \mathrm{C}$ for $10 \mathrm{hrs}$. Different batches of metallic and semiconducting ferrocene filled SWCNT were annealed in vacuum at $600{ }^{\circ} \mathrm{C}$ for $12 \mathrm{hrs}$ and measured in a SQUID magnetometer MPMS-XL, with magnetic fields up to $7 \mathrm{~T}$ and sample temperatures from $5 \mathrm{~K}$ to $300 \mathrm{~K}$.

\section{RESULTS AND DISCUSSION}

The XMCD signal is defined as the difference between the XAS spectra measured at both circular polarizations $\left(\mu^{+}-\mu^{-}\right)$. We observe no XMCD signals at $0 \mathrm{~T}$ (not shown). The XAS and XMCD spectra for the metallicity sorted $\mathrm{FeCp}_{2} @ \mathrm{SWCNT}$ at the $\mathrm{L}_{2,3} \mathrm{Fe}$ edge are depicted in Fig. 2. The spectra were recorded at $5 \mathrm{~K}$ and at a magnetic field of $6 \mathrm{~T}$. For ease of comparison, the recorded spectra are normalized by the average area of the L edge XAS $\int\left(\mu^{+}+\mu^{-}\right) / 2$ and offsetted by 0.1 . The XAS response shows slight dependency on the light polarization, Fig. 2(a). The spectral shapes before annealing are in good agreement with those of ferrocene encapsulated in SWCNT, reported in the previous work on mixed ${ }^{31}$ and separated samples. ${ }^{41}$ The main spin-orbit splitting features $\mathrm{L}_{3}$ and $\mathrm{L}_{2}$ located at $710.3,712.5 \mathrm{eV}$, and 722.6, $724.9 \mathrm{eV}$, respectively, coincide with those characteristic to the molecular orbitals of ferrocene observed in solids. ${ }^{42}$ The feature at $709.5 \mathrm{eV}$ could be characteristic of encapsulated ferrocene, that was less significant but also observed in the previous work. ${ }^{31}$ This feature shows a slight difference between the semiconducting and the metallic samples, which can be attributed to two possible effects: (i) the encapsulated molecules interacting with different proportions of metallic and semiconducting nanotubes or (ii) the presence of $\mathrm{Fe}$ impurities in the samples. The corresponding XMCD are
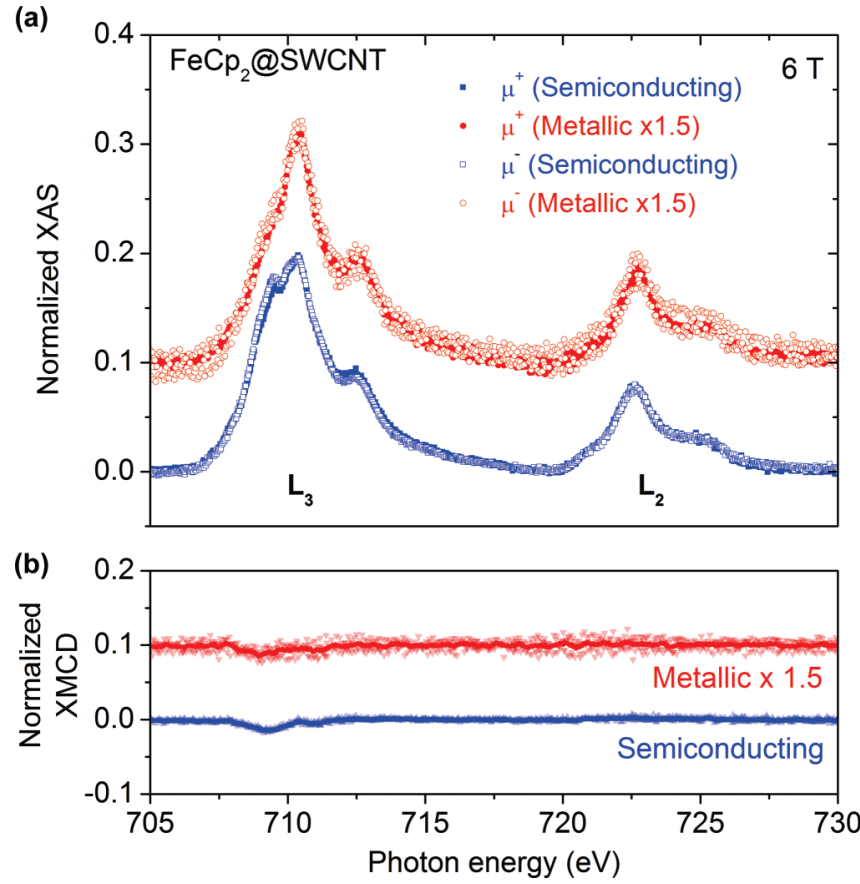

FIG. 2. (Color online) Observed XAS (a) and XMCD (b) response in the $\mathrm{L}_{2,3}$ edge of $\mathrm{Fe}$ for the metallic and the semiconducting FeCp $\mathrm{F}_{2} @$ SWCNT samples when a magnetic field of $6 \mathrm{~T}$ is applied to the sample at $5 \mathrm{~K}$. All the spectra are offsetted by 0.1 and normalized by $\int\left(\mu^{+}+\mu^{-}\right) / 2$.

depicted in Fig. 2(b). The main peak in the XMCD signal of FeCp $\mathrm{p}_{2} @ \mathrm{SWCNT}$ corresponds to the low energy band feature at $709.5 \mathrm{eV}$ in the XAS signal. The XMCD signal is larger for the semiconducting nanotubes than the metallic ones. No contribution from the $\mathrm{L}_{2}$ edge is observed.

Provided that all the XAS spectra were collected at the nearly identical experimental condition, except for the polarization of the light and magnetic field, the spectral intensity integrated over the $\mathrm{Fe} 2 \mathrm{p}$ edge is closely related to the concentration of $\mathrm{Fe}$ inside the nanotubes. A filling factor of $44 \%$ was first determined for a nonseparated SWCNT sample from XAS measurements at the C1s $\pi$ edge by M. Sauer et $a{ }^{41}{ }^{41}$ From this value, by considering the differences in the XAS spectral intensity integrated over the Fe edge, filling factors of $49 \%$ and $30 \%$ can be determined for the filled semiconducting and metallic samples, respectively. At room temperature no difference between $\mu^{+}$and $\mu^{-}$was observed for the semiconducting and metallic FeCp $\mathrm{p}_{2} @$ SWCNT, which means no XMCD response.

After annealing the FeCp $\mathrm{F}_{2} @ \mathrm{SWCNT}$ samples above $500{ }^{\circ} \mathrm{C}$ for several hours, the ferrocene inside the nanotubes decomposes and forms inner tubes and iron carbide $\mathrm{Fe}_{3} \mathrm{C}$ clusters (Fe@DWCNT), as proved by XAS and TEM studies (Fig. 1). In Fig. 3, the XAS and XMCD spectra for the Fe@DWCNT samples are shown. The spectra were recorded at $4 \mathrm{~T}$ and room temperature. The recorded spectra are normalized by the average area of the L edge XAS $\int\left(\mu^{+}+\mu^{-}\right) / 2$ and offset by 0.1 . The spectra are significantly altered in shape by annealing and composed of the two main skewed spin-orbit splitting peaks $L_{3}$ and $L_{2}$ located at 709.3 and $722.2 \mathrm{eV}$, respectively, 

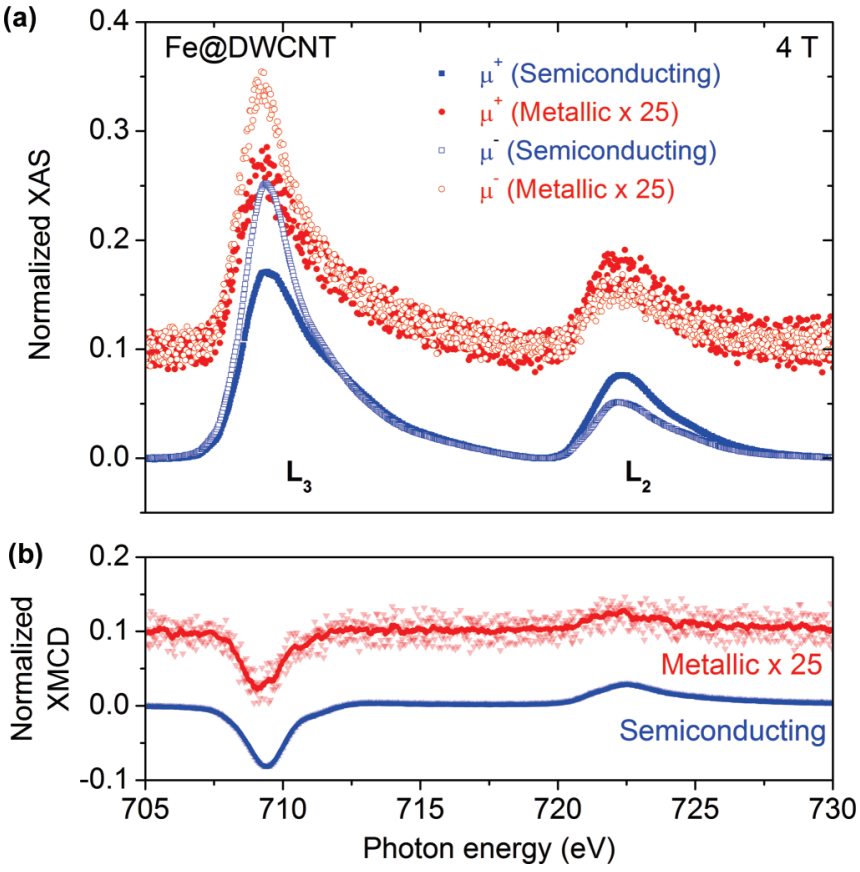

FIG. 3. (Color online) Observed XAS (a) and XMCD (b) response in the $\mathrm{L}_{2,3}$ edge of $\mathrm{Fe}$ for the metallic and the semiconducting Fe@DWCNT samples when a magnetic field of $4 \mathrm{~T}$ is applied to the sample at room temperature. All the spectra are offset by 0.1 and normalized by $\int\left(\mu^{+}+\mu^{-}\right) / 2$.

Fig. 3(a). From the spectral shapes and energies, these features can be assigned to metallic Fe. ${ }^{43,44}$

The effect of applying a magnetic field is more significant than in the samples before annealing, Fig. 3(a). The XMCD signal for the Fe@DWCNT is larger than that for the FeCp $\mathrm{p}_{2} @ \mathrm{SWCNT}$, Fig. 3(b). The formation of the cementite clusters enhances the magnetic response of the filled nanotubes. The difference in XAS intensity between the two different metallicity samples is greater for the Fe@DWCNT. This difference can be attributed to the formation of larger cementite clusters due to the higher filling factor in the semiconducting nanotubes.

The orbital $\left(m_{\text {orb }}\right)$ and spin $\left(m_{\text {spin }}\right)$ magnetic moments have been calculated from the XAS and XMCD spectra by using the sum rules: ${ }^{34,35}$

$$
m_{\text {orb }}=-\frac{4 q}{3 r}\left(10-n_{3 \mathrm{~d}}\right)
$$

and

$$
m_{\text {spin }}=-\frac{(6 p-4 q)}{r}\left(10-n_{3 \mathrm{~d}}\right)-7\left\langle T_{Z}\right\rangle,
$$

where $n_{3 \mathrm{~d}}$ is 6.61 corresponding to the $3 \mathrm{~d}$ electron occupation of Fe calculated theoretically; ${ }^{45} r$ is the integral of the XAS spectrum over the whole $\mathrm{L}_{2,3}$ edge [ Fig. 4(a)]; $p$ and $q$ are the integrals of the XMCD signal at the $\mathrm{L}_{3}$ edge and the whole $\mathrm{L}_{2,3}$ edge, respectively [Fig. 4(b)]. The expectation value of the magnetic dipole operator $\left\langle T_{Z}\right\rangle$ in the sum rules is neglected. ${ }^{46,47}$

The experimental spin $\left(\mathrm{m}_{S}\right)$ and orbital $\left(\mathrm{m}_{L}\right)$ magnetic moments of the metallicity sorted FeCp $\mathrm{p}_{2} @ \mathrm{SWCNT}$ samples are shown in Fig. 5 as a function of the applied magnetic field.
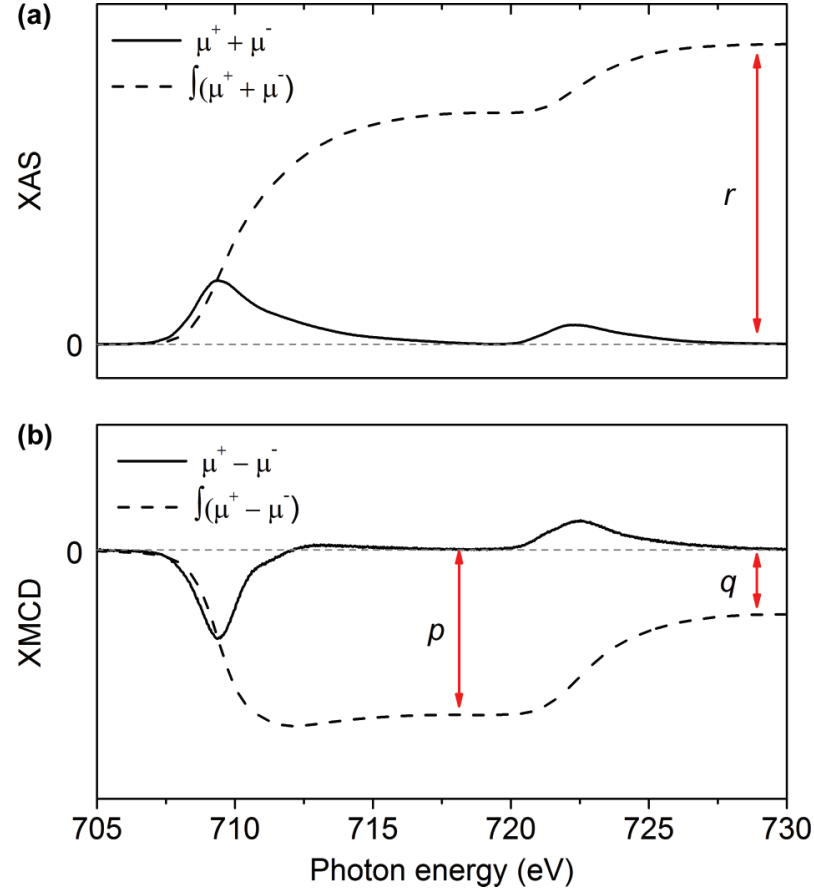

FIG. 4. (Color online) Definition of the integrals used to calculate the orbital [Eq. (1)] and spin [Eq. (2)] magnetic moments. (a) Total XAS and its integral in the whole $\mathrm{L}_{2,3}$ edge $(r)$; (b) XMCD response and its integrals in the $\mathrm{L}_{3}(p)$ and the whole $\mathrm{L}_{2,3}$ edge $(q)$.

The experimental data were fitted by a modified Langevin function:

$$
M(x)=M_{S, L}(\operatorname{coth}(x)-1 / x),
$$

where $M_{S, L}$ is the saturation magnetization of the spin (S) or the orbital (L) magnetic moments; $x=\mu_{c} H / k_{B} T$, in which $\mu_{c}$ is the uncompensated magnetic moment associated with the nanoparticle core, ${ }^{49} H$ is the applied magnetic field, $k_{B}$ is the Boltzmann constant, and $T$ is the temperature.

The saturation values $M_{L, S}$ obtained for the filled samples and the reference values for bulk iron are listed in Table I.

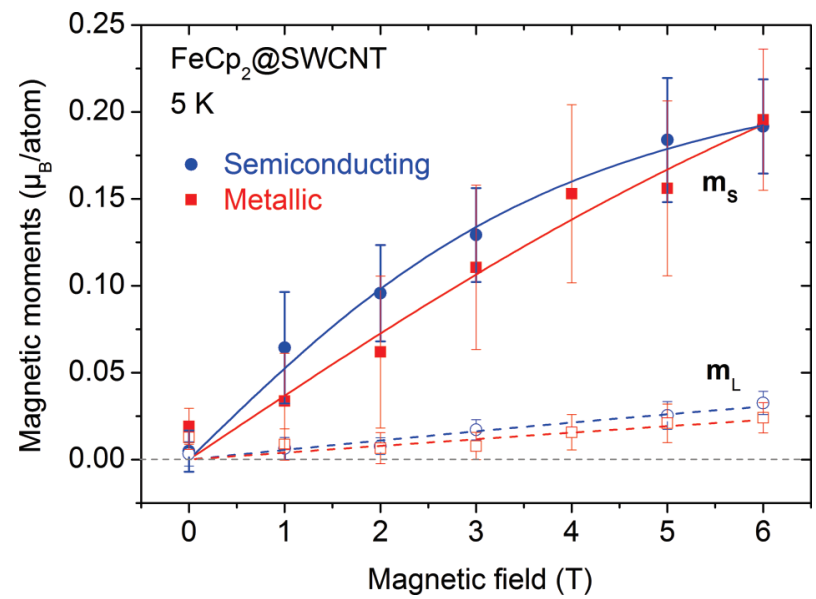

FIG. 5. (Color online) Spin and orbital magnetic moments of the metallic and semiconducting $\mathrm{FeCp}_{2} @ \mathrm{SWCNT}$. The data are fitted with the modified Langevin function. The measurements were done at $5 \mathrm{~K}$. 
TABLE I. Saturation values of the spin $\left(\mathrm{M}_{S}\right)$ and orbital $\left(\mathrm{M}_{L}\right)$ magnetic moments of pure $\mathrm{Fe}$ and fitting parameters, e.g., saturation magnetization $\left(\mathrm{M}_{S, L}\right)$ and uncompensated magnetic moment $\left(\mu_{C}\right)$ for the spin and orbital magnetic moments in $\mu_{B} /$ atom, of the metallic $(\mathrm{M})$ and semiconducting (SC) FeCp $2 @$ SWCNT and Fe@DWCNT

\begin{tabular}{|c|c|c|c|c|c|c|}
\hline & \multirow[b]{2}{*}{$\mathrm{Fe}$} & \multirow{2}{*}{$\begin{array}{l}\text { Fitting } \\
\text { param. }\end{array}$} & \multicolumn{2}{|c|}{$\mathrm{FeCp}_{2} @ \mathrm{SWCNT}$} & \multicolumn{2}{|c|}{ Fe@DWCNT } \\
\hline & & & $\mathrm{SC}$ & M & $\mathrm{SC}$ & M \\
\hline \multirow[t]{2}{*}{ Spin } & 1.98 (Ref. 43) & $M_{S}$ & 0.26 & 0.43 & 1.38 & 1.19 \\
\hline & & $\mu_{C}$ & 4.54 & 1.90 & 6552 & 2799 \\
\hline \multirow[t]{2}{*}{ Orbital } & 0.086 (Ref. 43) & $M_{L}$ & & & 0.086 & 0.084 \\
\hline & & $\mu_{C}$ & & & 8062 & 4343 \\
\hline
\end{tabular}

The saturation value of the total angular moment reported for cementite $\left(1.8 \mu_{B} \text { /atom }\right)^{48}$ is lower only by $\sim 10 \%$ than the total magnetic moment for iron $\left(2.0 \mu_{B} /\right.$ atom). ${ }^{43}$ As no spin and orbital magnetic moments of cementite have been reported, we compare our $M_{L, S}$ data to the bulk iron values. The obtained saturation values for the spin magnetic moments in the semiconducting $\left(M_{S}=0.26 \mu_{B} /\right.$ atom $)$ and metallic $\left(M_{S}=0.43 \mu_{B} /\right.$ atom $)$ FeCp $2 @$ SWCNT samples are lower by $\sim 90 \%$ and $\sim 80 \%$, respectively, than the value expected for bulk iron. ${ }^{43}$ The reduced spin magnetic moment in FeCp $\mathrm{p}_{2} @ \mathrm{SWCNT}$ could be due to poor interactions between the adjacent $\mathrm{Fe}$ atoms, resulting in a low $\left(\mathrm{m}_{S}\right)$ ordering of the spins. We could not obtain the $M_{L}$ values for $\mathrm{FeCp}_{2} @ \mathrm{SWCNT}$ due to the small $\mathrm{m}_{L}$ almost linearly dependent on the magnetic field. This paramagnetic behavior at high magnetic fields is observed for the $\mathrm{m}_{S}$ too, and indicates low magnetic permeability of iron in $\mathrm{FeCp}_{2} @ \mathrm{SWCNT}$.

By the transformation of the ferrocene to one-dimensional $\mathrm{Fe}_{3} \mathrm{C}$ inside the nanotubes, the iron $3 \mathrm{~d}$ magnetic moments increase considerably, Fig. 6. The experimental data was fitted by a modified Langevin function [Eq. (3)].

The saturation values of the spin magnetic moment for the semiconducting and metallic Fe@DWCNT samples are $M_{S}=1.38$ and $1.19 \mu_{B}$ /atom, respectively (Fig. 6). These values are lower by $\sim 30 \%$ and $\sim 40 \%$, respectively, than the expected cementite values. The enhanced saturation value

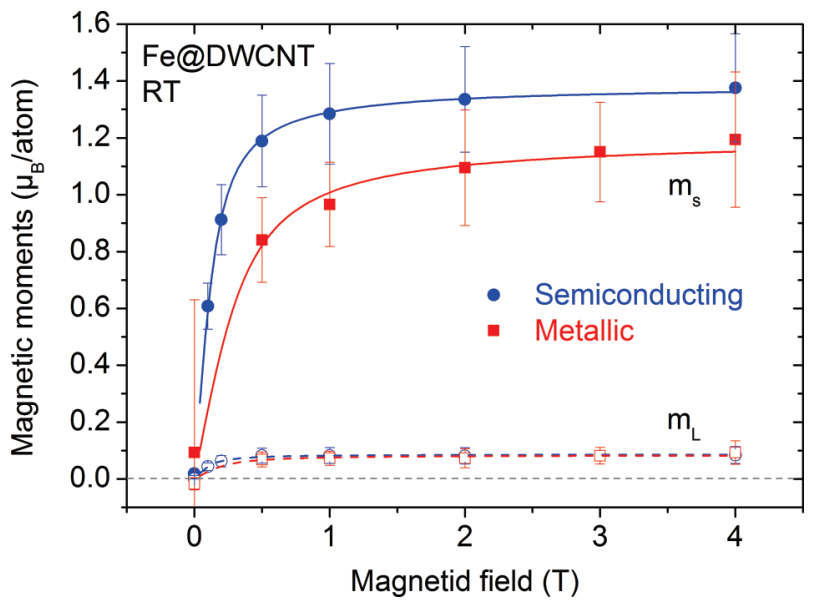

FIG. 6. (Color online) Spin and orbital magnetic moments of the metallic and semiconducting Fe@DWCNT. The data are fitted with the modified Langevin function. The measurements were done at $300 \mathrm{~K}$. after annealing means that the spins get more ordered as the chemical status of the filling changes from ferrocene to the 1D cementite cluster. The difference between the two metallicity Fe@DWCNT samples could be originating from the cluster size due to the filling degree and/or from the metallicity. Since the quantity of available iron atoms defines the mean length of the iron cluster formed inside the nanotube, the higher filling of the semiconducting sample leads to the formation of larger iron clusters which tend to have a greater spin ordering due to the size effect. ${ }^{50}$ Alternatively, the enhanced metallicity of the SWCNT could also reduce the spin ordering in the encapsulated metal via scattering by conduction electrons. The saturation value for the orbital magnetic moments of iron in Fe@DWCNT are $M_{L}=0.086$ and $0.084 \mu_{B}$ /atom for semiconducting and metallic, respectively, comparable to those reported for pure $\mathrm{Fe}\left(0.086 \mu_{B} / \text { atom }\right)^{43}$ and the corresponding cementite value, with a very small difference between the two metallicity samples.

Another batch of metallicity sorted FeCp $\mathrm{p}_{2} @ \mathrm{SWCNT}$ and Fe@DWCNT were measured by SQUID in order to compare the "local" and the "bulk" character of the magnetic properties of these materials. The former refers to the magnetic moments strongly localised at the iron atoms while the latter stands for the sum of all magnetisms obtained by SQUID. The filling inspected by Raman spectroscopy is $\sim 34 \%$ and $\sim 8 \%$ for the metallic and semiconducting samples, respectively, in this case.

Figure 7 depicts the room temperature magnetization curves for the semiconducting and metallic samples measured at room temperature. The data for the FeCp $\mathrm{F}_{2} @ \mathrm{SWCNT}$ and Fe@DWCNT are the magnetizations subtracted by the reference data. Both the empty SWCNT samples exhibit diamagnetism, Fig. 7(a). This changes to paramagnetism in the semiconducting $\mathrm{FeCp}_{2} @ \mathrm{SWCNT}$ while the magnetic response of the metallic FeCp $\mathrm{p}_{2} @$ SWCNT remains diamagnetic, Fig. 7(a). The drastic change in the semiconducting sample can be attributed to the weak interaction between the SWCNT and $\mathrm{FeCp}_{2}$, and the low filling degree. When both aspects are present, the $\mathrm{FeCp}_{2}$ molecules are electronically more isolated and possibly free to rotate inside the tubes. Under a magnetic field, these molecular magnets respond to exhibit the paramagnetic behavior. In the case of the metallic sample the interaction between the $\mathrm{FeCp}_{2}$ and SWCNT is stronger due to the higher metallicity, as well as between the adjacent $\mathrm{FeCp}_{2}$ molecules due to the higher filling degree. This leads to more delocalized electrons over the sample, which can result in the enhanced diamagnetism as observed in the right panel in Fig. 7(a). 


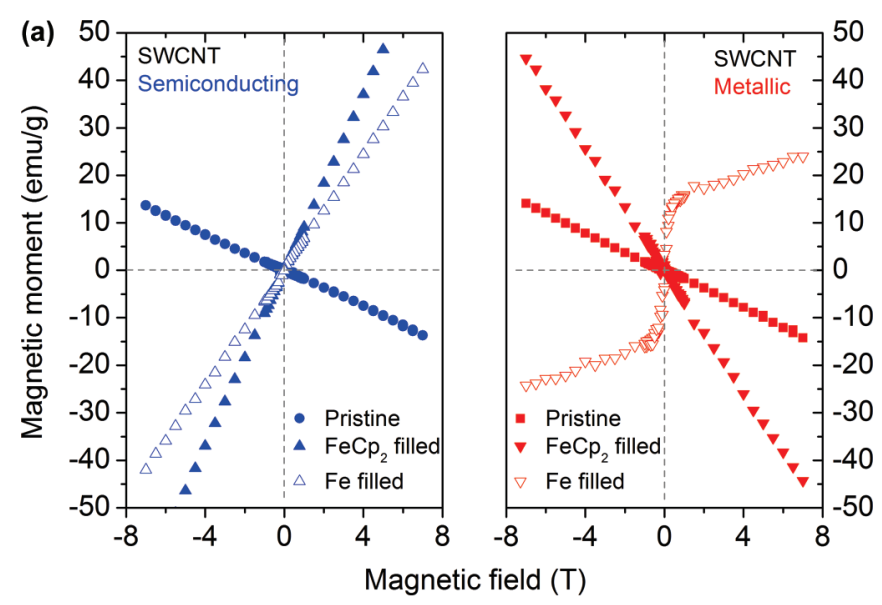

(b)

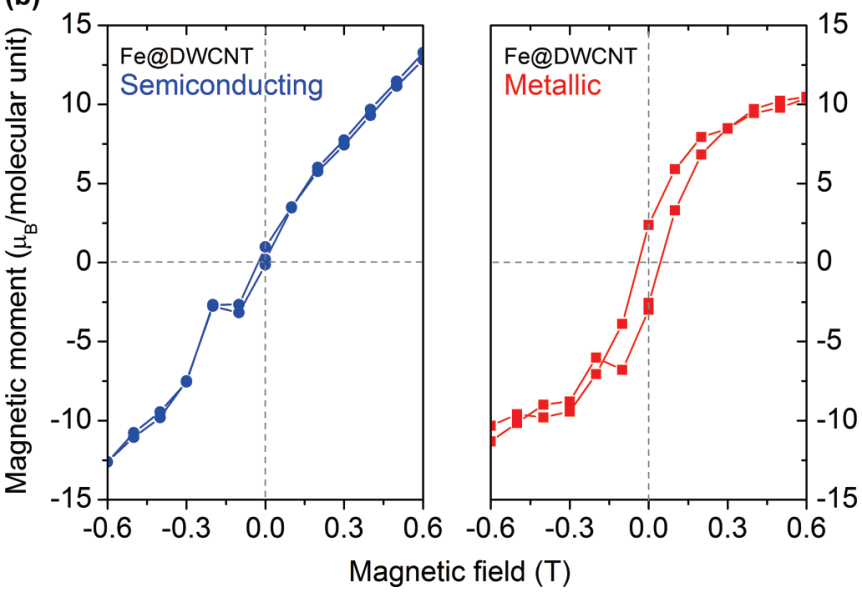

FIG. 7. (Color online) Total magnetic moment measured by SQUID. (a) For pristine semiconducting and metallic SWCNT, FeCp $\mathrm{p}_{2} @$ SWCNT and Fe@DWCNT; (b) in the low magnetic field region for semiconducting and metallic Fe@DWCNT. The magnetic moments were calculated per molecular unit with one Fe atom (b). The diamagnetic background of the pristine nanotubes were subtracted from the data of the $\mathrm{FeCp}_{2}$ and Fe filled nanotubes. The measurements were done at $300 \mathrm{~K}$.

After the transformation to Fe@DWCNT, the semiconducting sample stays paramagnetic. For the metallic sample, which has a filling degree higher by $23 \%$ than the semiconducting sample, a ferromagnetic behavior is evident with a coercivity of $40 \mathrm{mT}$, Fig. 7(b). This ferromagnetism is thought to be enhanced due to the larger cementite cluster size and possibly the strong metallic character. The small coercivity value for the semiconducting sample means that the cementite nanoparticles hardly interact with each other and the spin alignment is poor owing to the smaller cluster size. Note that the magnetic moments obtained by SQUID are much larger than those obtained by XMCD, due to the "in bulk" character of the measurements in which the contributions of electron spins in the conduction band are observed. Hence, the remarkable differences between the filled metallic and semiconducting samples observed by SQUID are associated with the delocalized magnetisms which should be more sensitive to changes in SWCNT metallicity.

\section{CONCLUSION}

The orbital and spin magnetic moments of the iron $3 \mathrm{~d}$ states in $\mathrm{FeCp}_{2} @$ SWCNT and Fe@DWCNT have been studied by XMCD. Signatures for the intermolecular interactions have been observed. The both orbital and spin magnetic moments are found to be paramagnetic and larger in the filled semiconducting SWCNT than in the filled metallic nanotubes. This can be attributed to the difference in iron cluster size that are larger in the semiconducting tubes due to the higher filling. Considerable differences between the filled metallic and semiconducting nanotubes have been observed by SQUID. The ferromagnetism observed in the metallic Fe@DWCNT and the paramagnetism in the filled semiconducting tubes can be explained in accordance with the differences in nanotube metallicity and cluster size. The delocalized and non-iron magnetic polarizations contribute significantly to the magnetic behavior of these nanocomposites.

\section{ACKNOWLEDGMENTS}

We acknowledge the Helmholtz-Zentrum Berlin-Electron storage ring BESSY II for the provision of synchrotron radiation at beamline UE46/PGM-1 and would like to thank Enrico Schierle for technical assistance. This work was supported by the Austrian Science Funds (FWF), project P621333-N20, and receiving funding from the European Community's Seventh Framework Programme (FP7/2007-2013) under Grant No. 226716. P.A. was supported by a Marie Curie Intra European Fellowship within the 7th European Community Framework Programme.
*Corresponding author: antonio.briones@univie.ac.at

${ }^{1}$ H. Ajiki and T. Ando, J. Phys. Soc. Jpn. 62, 2470 (1993).

${ }^{2}$ K. Lipert, F. Kretzschmar, M. Ritschel, A. Leonhardt, R. Klingeler, and B. Bchner, J. Appl. Phys. 105, 063906 (2009).

${ }^{3}$ J. Kono, R. J. Nicholas, and S. Roche, in Carbon Nanotubes: Advanced Topics in the Synthesis, Structure, Properties, and Applications, edited by A. Jorio, G. Dresselhaus, and M. S. Dresselhaus (Springer, Berlin, Heidelberg, 2008), p. 393.

${ }^{4}$ A. Hirsch, Angew. Chem., Int. Ed. 41, 1853 (2002).

${ }^{5}$ M. Monthioux, Carbon 40, 1809 (2002).
${ }^{6}$ B. W. Smith, M. Monthioux, and D. E. Luzzi, Nature (London) 396, 323 (1998).

${ }^{7}$ J. Sloan, S. Friedrichs, R. R. Meyer, A. I. Kirkland, J. L. Hutchison, and M. L. H. Green, Inorg. Chim. Acta 330, 1 (2002).

${ }^{8}$ R. Kitaura and H. Shinohara, Jpn. J. Appl. Phys. 46, 881 (2007).

${ }^{9}$ L. J. Li, A. N. Khlobystov, J. G. Wiltshire, G. A. D. Briggs, and R. J. Nicholas, Nat. Mater. 4, 481 (2005).

${ }^{10}$ R. Kitaura, H. Okimoto, H. Shinohara, T. Nakamura, and H. Osawa, Phys. Rev. B 76, 172409 (2007). 
${ }^{11}$ Y. Li, T. Kaneko, T. Ogawa, M. Takahashi, and R. Hatakeyama, Chem. Commun. 254 (2007).

${ }^{12}$ R. Kitaura, D. Ogawa, K. Kobayashi, T. Saito, S. Ohshima, T. Nakamura, H. Yoshikawa, K. Awaga, and H. Shinohara, Nano Research 1, 152 (2008).

${ }^{13}$ Y. Li, T. Kaneko, T. Ogawa, M. Takahashi, and R. Hatakeyama, Jpn. J. Appl. Phys. 47, 2048 (2008).

${ }^{14}$ H. Shiozawa, T. Pichler, A. Gruneis, R. Pfeiffer, H. Kuzmany, Z. Liu, K. Suenaga, and H. Kataura, Adv. Mater. 20, 1443 (2008).

${ }^{15}$ H. Shiozawa, T. Pichler, C. Kramberger, M. Rummeli, D. Batchelor, Z. Liu, K. Suenaga, H. Kataura, and S. R. P. Silva, Phys. Rev. Lett. 102, 046804 (2009).

${ }^{16}$ M. Koshino, Y. Niimi, E. Nakamura, H. Kataura, T. Okazaki, K. Suenaga, and S. Iijima, Nat. Chem. 2, 117 (2010).

${ }^{17}$ H. Shiozawa, C. Kramberger, R. Pfeiffer, H. Kuzmany, T. Pichler, Z. Liu, K. Suenaga, H. Kataura, and S. R. P. Silva, Adv. Mater. 22, 3685 (2010).

${ }^{18}$ F. X. Geng and H. T. Cong, Physica B-Condensed Matter 382, 300 (2006).

${ }^{19}$ A. Vyalikh, A. U. B. Wolter, S. Dampel, D. Haase, M. Ritschel, A. Leonhardt, H. J. Grafe, A. Taylor, K. Kramer, B. Buchner, and R. Klingeler, Nanomedicine 3, 321 (2008).

${ }^{20}$ N. Grobert, W. K. Hsu, Y. Q. Zhu, J. P. Hare, H. W. Kroto, D. R. M. Walton, M. Terrones, H. Terrones, P. Redlich, M. Ruhle, R. Escudero, and F. Morales, Appl. Phys. Lett. 75, 3363 (1999).

${ }^{21}$ G. E. Grechnev, V. A. Desnenko, A. V. Fedorchenko, A. S. Panfilov, Y. A. Kolesnichenko, L. Y. Matzui, M. I. Grybova, Y. I. Prylutskyy, U. Ritter, and P. Scharff, Low Temp. Phys. 36, 1086 (2010).

${ }^{22}$ U. Ritter, P. Scharff, G. E. Grechnev, V. A. Desnenko, A. V. Fedorchenko, A. S. Panfilov, Y. I. Prylutskyy, and Y. A. Kolesnichenko, Carbon 49, 4443 (2011).

${ }^{23}$ D. Hisada, Y. Fujiwara, H. Sato, M. Jimbo, T. Kobayashi, and K. Hata, J. Magn. Magn. Mater. 323, 3184 (2011).

${ }^{24}$ B. Bittova, J. P. Vejpravova, M. Kalbac, S. Burianova, A. Mantlikova, S. Danis, and S. Doyle, J. Phys. Chem. C 115, 17303 (2011).

${ }^{25}$ E. Borowiak-Palen, E. Mendoza, A. Bachmatiuk, M. H. Rummeli, T. Gemming, J. Nogues, V. Skumryev, R. J. Kalenczuk, T. Pichler, and S. R. P. Silva, Chem. Phys. Lett. 421, 129 (2006).

${ }^{26}$ Y.-J. Kang, J. Choi, C.-Y. Moon, and K. J. Chang, Phys. Rev. B 71, 115441 (2005).

${ }^{27}$ J. Wang, C. Jo, and R. Wu, Appl. Phys. Lett. 92, 032507 (2008).

${ }^{28}$ H. Shinohara, Rep. Prog. Phys. 63, 843 (2000).

${ }^{29}$ P. Ayala, R. Kitaura, C. Kramberger, H. Shiozawa, N. Imazu, K. Kobayashi, D. Mowbray, P. Hoffmann, H. Shinohara, and T. Pichler, Materials Express 1, 30 (2011).

${ }^{30}$ P. Ayala, R. Kitaura, R. Nakanishi, H. Shiozawa, D. Ogawa, P. Hoffmann, H. Shinohara, and T. Pichler, Phys. Rev. B 83, 085407 (2011).
${ }^{31}$ H. Shiozawa, T. Pichler, C. Kramberger, A. Gruneis, M. Knupfer, B. Buchner, V. Zolyomi, J. Koltai, J. Kurti, D. Batchelor, and H. Kataura, Phys. Rev. B 77, 153402 (2008).

${ }^{32}$ J. Stohr, J. Magn. Magn. Mater. 200, 470 (1999).

${ }^{33}$ H. Shiozawa, T. Miyahara, K. Obu, Y. Takayama, H. Ishii, T. D. Matsuda, H. Sugawara, H. Sato, T. Muro, and Y. Saitoh, J. Phys. Soc. Jpn. 72, 2079 (2003).

${ }^{34}$ B. T. Thole, P. Carra, F. Sette, and G. van der Laan, Phys. Rev. Lett. 68, 1943 (1992).

${ }^{35}$ P. Carra, B. T. Thole, M. Altarelli, and X. D. Wang, Phys. Rev. Lett. 70, 694 (1993).

${ }^{36}$ K. Yanagi, H. Udoguchi, S. Sagitani, Y. Oshima, T. Takenobu, H. Kataura, T. Ishida, K. Matsuda, and Y. Maniwa, ACS Nano 4, 4027 (2010).

${ }^{37}$ H. Ishii, H. Kataura, H. Shiozawa, H. Yoshioka, H. Otsubo, Y. Takayama, T. Miyahara, S. Suzuki, Y. Achiba, M. Nakatake, T. Narimura, M. Higashiguchi, K. Shimada, H. Namatame, and M. Taniguchi, Nature (London) 426, 540 (2003).

${ }^{38}$ H. Rauf, T. Pichler, M. Knupfer, J. Fink, and H. Kataura, Phys. Rev. Lett. 93, 096805 (2004).

${ }^{39}$ P. Ayala, Y. Miyata, K. De Blauwe, H. Shiozawa, Y. Feng, K. Yanagi, C. Kramberger, S. R. P. Silva, R. Follath, H. Kataura, and T. Pichler, Phys. Rev. B 80, 205427 (2009).

${ }^{40}$ A. Briones, X. Liu, C. Kramberger, T. Saito, and T. Pichler, Phys. Status Solidi B 248, 2488 (2011).

${ }^{41}$ M. Sauer, H. Shiozawa, P. Ayala, G. Ruiz-Soria, X. Liu, A. Chernov, S. Krause, K. Yanagi, H. Kataura, and T. Pichler, Carbon 59, 237 (2013).

${ }^{42}$ A. Hitchcock, A. Wen, and E. Rhl, Chem. Phys. 147, 51 (1990).

${ }^{43}$ C. T. Chen, Y. U. Idzerda, H. J. Lin, N. V. Smith, G. Meigs, E. Chaban, G. H. Ho, E. Pellegrin, and F. Sette, Phys. Rev. Lett. 75, 152 (1995).

${ }^{44}$ J. T. Lau, A. Fohlisch, R. Nietubyc, M. Reif, and W. Wurth, Phys. Rev. Lett. 89, 057201 (2002).

${ }^{45}$ G. Y. Guo, H. Ebert, W. M. Temmerman, and P. J. Durham, Phys. Rev. B 50, 3861 (1994).

${ }^{46}$ M. Komelj, C. Ederer, J. W. Davenport, and M. Fähnle, Phys. Rev. B 66, 140407 (2002).

${ }^{47}$ S. Peredkov, M. Neeb, W. Eberhardt, J. Meyer, M. Tombers, H. Kampschulte, and G. Niedner-Schatteburg, Phys. Rev. Lett. 107, 233401 (2011)

${ }^{48}$ E. Duman, M. Acet, E. F. Wassermann, J. P. Itié, F. Baudelet, O. Mathon, and S. Pascarelli, Phys. Rev. Lett. 94, 075502 (2005).

${ }^{49}$ S. Kilcoyne and R. Cywinski, J. Magn. Magn. Mater. 140-144, 1466 (1995).

${ }^{50}$ M. Niemeyer, K. Hirsch, V. Zamudio-Bayer, A. Langenberg, M. Vogel, M. Kossick, C. Ebrecht, K. Egashira, A. Terasaki, T. Moller, B. V. Issendorff, and J. T. Lau, Phys. Rev. Lett. 108, 057201 (2012). 